\title{
Microprobe Endstation at SXRMB of Canadian Light Source: Introduction and Applications
}

\author{
Q.F. Xiao $^{1 *}$, Y.F. Hu${ }^{1}$ and T.K. Sham ${ }^{2}$ \\ 1. Canadian Light Source Inc., Saskatoon, Canada. \\ 2. Department of Chemistry, University of Western, London, Canada \\ * Corresponding author email: qunfeng.xiao@lightsource.ca.
}

Mapping of chemical element's distribution and Microprobe XAFS are the popular method to meet fundamental researches and industrial applications. Kirkpatrick-Baez (K-B) mirrors are normally utilized to realize the micro-focusing, commonly for energies above $4000 \mathrm{eV}$. At SXRMB, the microprobe station was designed to operate under vacuum, with a focus on applications in the challenging medium-energy region (2-4 keV). It uses a bending magnet source, covers an energy range of $1.7-10.0 \mathrm{keV}$ and uses a pair of K-B mirrors, focusing the collimated beam to a spot size of $10 \mu \mathrm{m} \times 10 \mu \mathrm{m}$ with $10^{8}-10^{9}$ photons/100mA/Sec flux [1]. A high resolution and large area CCD camera is equipped to obtain optical image of sample. A 4-element Si drift detector is used for mapping in fluorescence mode and TEY mapping is also possible.

The SXRMB microprobe station has been open to general users with its application focusing on main group elements such as $\mathrm{Si}, \mathrm{P}, \mathrm{S}$, combined with metals, such as $\mathrm{Ca}, \mathrm{Cr}$ and $\mathrm{Fe}$ [1]. One of the research areas is the microanalysis of phosphorus in soil and agriculture. The legacy phosphorus in soil has been studied for its potential utilization as secondary $\mathrm{P}$ resource [2]. The $\mu$-XRF maps of two soils, with and without $\mathrm{P}$ fertilization, from long term plots (Fig. 1) display the presence of minute $\mathrm{P}$ hot spots disseminated in a low-concentration diffuse background. The overlapping discrete hot spots of $\mathrm{P}$ and $\mathrm{Ca}$ agree well with the good correlation of $\mathrm{P}$ with $\mathrm{Ca}$, but the poor correlation with other elements such as $\mathrm{Fe}$, Al and Si. Further $\mu$-XANES spectra were collected at specific points of interest on these soils (Fig 1c). These spectra, with features on peaks 3 and 4, matched well with the bulk-XANES spectrum of HAP. Therefore, the micron-scale observations presented in this study visually confirmed the dominance of hydroxylapatite (HAP) at P hot spots of the 2015 soils. Additionally, the pre-edge peak 1 in $\mu$-XANES spectra of the 2015 soils indicated the presence of Fe-P, particularly for the nitrogen-potassium (NK) soil, which did not appear in the bulk-XANES spectra. The presence of Fe-P seems reasonable given the wide distribution of $\mathrm{Fe}$ (hydr) oxides as $\mathrm{P}$ sorbents in the 2015 soils as indicated by the diffusion of Fe signals by $\mu$-XRF (Fig. 1a and b).

The Sulfur chemical states of rat brain tissues have been measured for the research of brain ischemia (stroke) [1]. The brain tissue excited with different energies across the S K-edge, is shown in Fig. 2. The bright field image (Fig. 2a) of the unstrained rat brain tissue section highlights hippocampal grey matter (red asterisk) and corpus callosum white matter (blue asterisk). Micro-XAS spectra (Fig. 2b) collected from these regions highlight a prominent absorption feature (position 1), which is due to low oxidation state sulfur species (thiols and disulfides) present in white matter and grey matter. However, the grey matter contains a stronger absorption edge due to sulfonic acids (position 2) whereas white matter contains a stronger absorption edge due to sulfate esters (position 3). Strong variation in $\mathrm{S}$ fluorescence occurs across the tissue sample, when the incident beam energy is set to either position 1, 2 or 3, and reflects differential distribution within white matter and grey matter of low oxidation state sulfur species, sulfonic acids (taurine) and sulfate esters (sulfatide lipids in the myelin sheath), respectively. 
These two examples have shown that the SXRMB microprobe station with beam size of $10 \mu \mathrm{m} \times 10 \mu \mathrm{m}$ and beam flux of $10^{8}$ photons $/ 100 \mathrm{~mA} / \mathrm{Sec}$ and optimized for medium energy is a very useful tool for elemental mapping and $\mu$-XAS analysis of soil and biological samples with relative low concentration of $\mathrm{P}, \mathrm{S}$ and $\mathrm{Ca}$; and it is widely used in other research areas, such as catalysis and cultural and heritage science [3].

References:

[1] Qunfeng Xiao et al, J. Synchrotron Rad. (2017). 24, 333-337.

[2] Jin Liu et al, Scientific Reports, 7, (2017), p. 15354.

[3] M.S. Kozchuk et al, Herit Sci (2018) 6:12

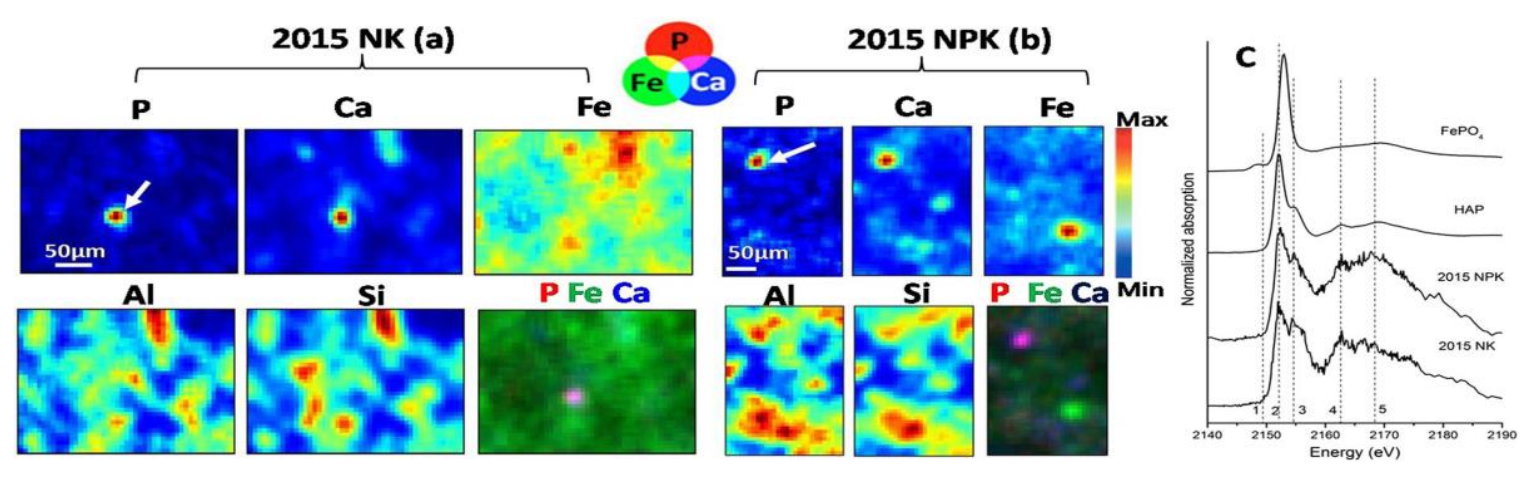

Figure 1. Distribution of $\mathrm{P}, \mathrm{Fe}, \mathrm{Al}, \mathrm{Ca}$, and $\mathrm{Si}$ in soils sampled in 2015 from the long-term plots without (NK, a) and with (NPK, b) 27 years of P fertilization by $\mu$-XRF mapping and P speciation at the selected hop spots probed by $\mu$-XANES (c). Individual signals of each element are shown with concentration proportional to brightness and RGB composite map of $\mathrm{P}, \mathrm{Fe}$ and $\mathrm{Ca}$ are also exhibited. Spectral peaks of interest are marked by vertical dashed lines and labeled from1 to 5: peak 1, Fe- P; peak 2, main peak; peak 3 and 4, Ca-P; peak 5, oxygen oscillation.
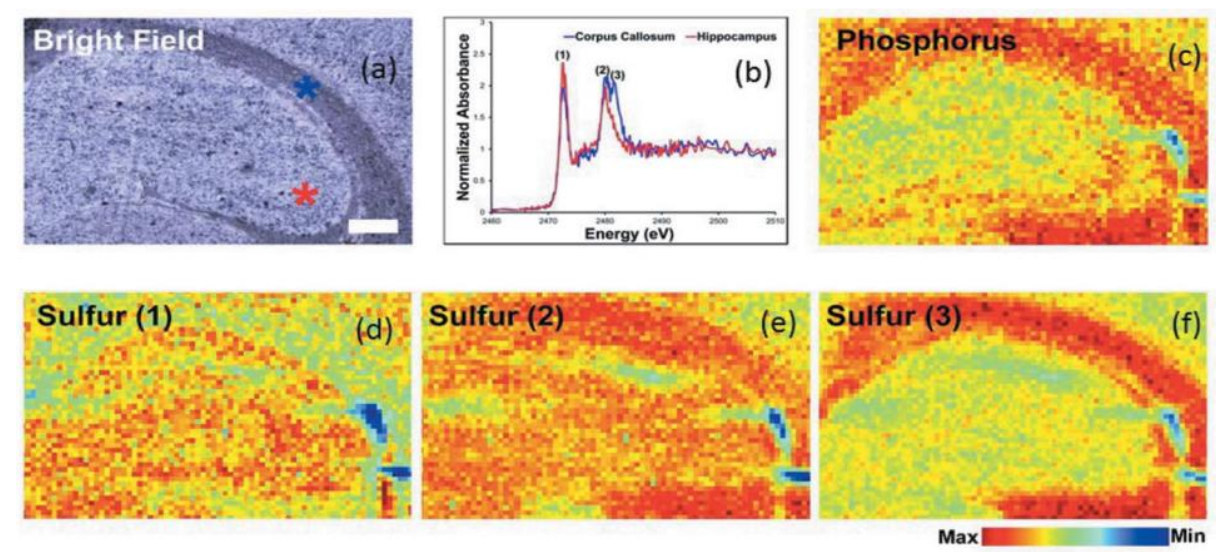

Figure 2. Chemically specific sulfur imaging of brain tissue. Bright field image (a) shows white matter (blue asterisk) and grey matter (red asterisk). (b) Corresponding sulfur micro-XAS spectra are shown in matching colours. Imaging was performed at incident energies of: (c) $2154 \mathrm{eV}$ for phosphorus, (d) 473.3 $\mathrm{eV}$ for low oxidation state sulfur, thiols and disulfides, (e) $2481 \mathrm{eV}$ for sulfonic acids and ( f) $2482.6 \mathrm{eV}$ for sulfates. Scale bar $=500 \mu \mathrm{m}$. 